\title{
ON CONTIGUOUS CONGRUENT SETS IN EUCLIDEAN SPACE
}

\author{
CHARLES J. A. HALBERG, JR., EUGENE LEVIN, AND E. G. STRAUS
}

1. Introduction. It is well-known that a circle can be surrounded by six translates of itself in such a way that the translates are each tangent to the original circle, are nonoverlapping, and their union encloses the original circle. In this note we extend this result in several directions. In $\$ 2$ we show that a very wide class of bounded sets in $E^{2}$ have a similar property. To keep the proof from being overly complicated we limit ourselves to the proof that this property is possessed by all sets consisting of the closed interior of a Jordan curve.

In $\S 3$ we consider higher dimensional bodies and show that the number of nonoverlapping spheres which can touch a given sphere serves as a lower bound of the number of nonoverlapping reflections in hyperplanes which can touch any given body. We also give some crude estimates of this number.

\section{We shall have need of the following definitions.}

Definition 1. Two sets are contiguous if their intersection is nonempty and is equal to the intersection of their frontiers.

Definition 2. A set $A$ encloses a set $B$ if every unbounded connected set which intersects $B$ also intersects $A$.

The principal result of this section is stated in the following theorem.

THEOREM. Let $S$ be the closed interior of a Jordan curve. Then for any direction $\theta$ there exist six translates of $S$ with the following properties:

(1) One translate is in the direction $\theta$.

(2) Each translate is contiguous to $S$.

(3) No two translates have interior points in common.

(4) Each translate is contiguous to two others.

(5) The union of the six translates encloses $S$.

We shall now give a sequence of lemmas designed to facilitate the proof of this theorem.

Let a rectangular coordinate system be established with the origin at an interior point of $S$. The image of $S$ under the translation which

Presented to the Society January 28, 1958; received by the editors June 23, 1958. 
moves the origin to a point $p=(x, y)$ will be denoted by $S(p)$ or, if coordinates are essential to the argument, by $S(x, y)$.

Lemma 1. If $A$ is any connected set such that there exist points $p$ and $q$ of $A$ for which $S(p) \cap S$ is null and $S(q) \cap S$ is non-null, then there exists a point $r$ of $A$ such that $S(r)$ is contiguous to $S$.

Proof. Assume that there does not exist a point $r$ of $A$ such that $S(r)$ is contiguous to $S$. Let $U$ and $V$ respectively, be the set of points $p \in A$ for which $S(p) \cap S$ is null and the set of points $p \in A$ for which $S(p) \cap S$ is non-null. The sets $U$ and $V$ are non-null by hypothesis and it is clear that their union is $A$. We shall now show that $U$ and $V$ are relatively open in $A$, which will contradict the assumption that $A$ is connected.

Let $u$ be any point of $U$. Since $S$, and therefore $S(u)$, is compact, and $S \cap S(u)$ is null, it follows that there is a positive distance $\delta$ between the two sets. It follows immediately that $U$ is open, since if $u^{\prime}$ is any point of a circular neighborhood of $u$ with radius $\delta / 2$, then $S \cap S\left(u^{\prime}\right)$ is null. Now let $v$ be any point of $V$. From the definition of $V$ and our hypothesis we are forced to conclude that there exists an interior point of $S \cap S(v)$. But this implies that $v$ is an interior point of $V$, whence $V$ is open. This completes the proof of our lemma.

Let $W$ denote the set of points $p$ for which $S(p)$ is contiguous to $S$. It is an immediate consequence of Lemma 1 that $W$ encloses $S$. It is also easy to prove that $W$ is symmetric with respect to the origin.

We shall let $E_{W}$ denote the set of points enclosed by $W$.

\section{Lemma 2. The set $E_{W}$ is compact.}

Proof. Since $E_{W}$ is clearly bounded, it suffices to show that it is closed. To this end let $p$ be any point of the complement of $E_{W}$. There exists an unbounded connected set $G$ containing $p$ such that the intersection of $G$ with $W$ is null. Since $G$ obviously contains a point $q$ such that $S(q) \cap S$ is null we see that $S(p) \cap S$ is null. For if this were not so it would follow from Lemma 1 that $G \cap W$ is non-null. Thus, since $S(p)$ and $S$ are a positive distance apart, it follows that there exists a neighborhood of $p$, such that if $p^{\prime}$ is any point of this neighborhood then $S\left(p^{\prime}\right) \cap S$ is null. We therefore conclude that the complement of $E_{W}$ is open, whence $E_{W}$ is closed.

Let $I$ denote the set of points $p$ such that $S(p) \cap S$ is non-null. It is not difficult to show that $I$ is contained in $E_{W}$, but in general is unequal to $E_{W}$, and that $W$ contains the boundary of $E_{W}$, but in general is not equal to it.

Lemma 3. $E_{W}$ is a continuum. 
Proof. We need only prove that $E_{W}$ is connected. We first prove that $I$ is connected.

Suppose that $(x, y) \in I$. Then there exists a point $\left(x_{0}, y_{0}\right) \in S(x, y)$ $\cap S$. (Note that this implies that $\left(x_{0}-x, y_{0}-y\right) \in S$.) It is obvious that $S\left(x-x_{0}, y-y_{0}\right)$ is connected and that it must contain the points $(x, y)$ and $(0,0)$. Both $S$ and $S\left(x-x_{0}, y-y_{0}\right)$ are contained in $I$. We now associate with $(x, y)$ the set $C_{x y}=S\left(x-x_{0}, y-y_{0}\right) \cup S$, which is a connected set contained in $I$ and containing $(x, y)$. The set $\mathrm{U}_{(x, y) \in I} C_{x y}$ is a connected set since it is the union of a set of connected sets each of which contains $S$. Thus, since $\bigcup_{(x, y) \in I} C_{x y}$ is clearly equal to $I$, we have proved that $I$ is connected.

Now, to show that $E_{W}$ is connected, we let $(x, y)$ be any point of $E_{W}$ and associate with $(x, y)$ a set, in the following manner. If $(x, y) \in I$ we associate with $(x, y)$ the set $I$. If $(x, y) \notin I$ let $\left(x^{\prime}, y^{\prime}\right)$ be a point of $I$ which is closest to $(x, y)$. Such a point exists since $I$ is compact. We now associate with this $(x, y)$ the set consisting of the union of $I$ with the line segment from $(x, y)$ to $\left(x^{\prime}, y^{\prime}\right)$. The line segment belongs to $E_{W}$. For suppose there were a point $(a, b)$ belonging to the segment, but not contained in $E_{W}$. Then, by Lemma 1 , there exists a point of $W$ between $(x, y)$ and $(a, b)$. But, since it is clear that $W$ is contained in $I$, this contradicts the fact that there are no points belonging to $I$ that are closer to $(x, y)$ than $\left(x^{\prime}, y^{\prime}\right)$. We have thus associated with each point of $E_{W}$ a connected set which contains $I$ and is contained in $E_{W}$. It follows immediately that their union is connected and is $E_{W}$.

Lemma 4. The complement of $E_{W}$ is a generalized continuum (i.e. a locally compact, connected set).

We need only prove that the complement of $E_{W}$ is connected since it is clearly locally compact. This connectedness follows immediately from the fact that all points of this complement can be joined to infinity.

Lemma 5. The boundary of $E_{W}$, bd $E_{W}$, is a continuum.

Proof. This is an immediate consequence of Lemmas 3 and 4 and a corollary to the Phragmen-Brouwer theorem which states "If the boundary $F$ of a complementary domain of a generalized continuum is bounded, $F$ is a continuum" $[1$, p. 106].

Lemma 6. If $S(p)$ and $S$ are contiguous, then bd $E_{W}$ and bd $E_{W}(p)$ have a non-null intersection.

Proof. Assume that $S$ and $S(p)$ are contiguous and that bd $E_{W}$ 
and bd $E_{W}(p)$ have a null intersection. If $q$ is a point of contiguity of $S$ and $S(p)$, then $q$ is an interior point of $E_{W}$ and $E_{W}(p)$, for it is clear that $S \subset$ int $E_{W}$ and $S(p) \subset$ int $E_{W}(p)$. Therefore int $E_{W}$ กint $E_{W}(p)$ is nonnull, and it follows, since $E_{W}$ is connected and ext $E_{W}(p)$ contains points of int $E_{W}$ that int $E_{W}$ has a nonnull intersection with bd $E_{W}(p)$. From this we infer that bd $E_{W}(p)$ is not connected, since

$$
\text { bd } E_{W}(p)=\left(\text { int } E_{W} \cap \operatorname{bd} E_{W}(p)\right) \cup\left(\operatorname{ext} E_{W} \cap \mathrm{bd} E_{W}(p)\right) .
$$

This gives the desired contradiction.

From Lemma 6 we conclude that, if $S(p)$ and $S$ are contiguous, then there exists a translate $S(q)$ which is contiguous to both $S$ and $S(p)$. The following lemma demonstrates that $p$ and $q$ cannot be colinear with the origin.

Definition 3. If $a, b \in \mathrm{bd} S$, then a spanning arc from $a$ to $b$ in $S$ is a closed arc $[a, b]$ such that $[a, b] \subset S$ and $[a, b] \cap b d S=\{a, b\}$.

Lemma 7. (a) Any two translates of $S$ in the same direction that are both contiguous with $S$ have interior points in common. (b) Any two translates of $S$ in opposite directions that are both contiguous with $S$ have no points in common.

Proof of (a). Assume that $S(p)$ and $S(q)$ are translates, in the same direction, of $S$ which are both contiguous to $S$ and that int $S(p)$ nint $S(q)$ is the null set. Let the positive $x$-axis be in the direction of the translations and let $L$ and $U$ be, respectively, the lower and upper lines of support of the configuration which are parallel to the $x$-axis. Assume that the distance from $p$ to the origin is greater than that from $q$ to the origin and let $r$ and $t$ be the points of $L \cap S(q)$ and $U \cap S(q)$, respectively, which have the largest abscissas. Then the union of a vertical ray emanating upward from $t$, a vertical ray emanating downward from $r$ and a spanning arc from $r$ to $t$ in $S(q)$ separates the plane so that $S$ and int $S(p)$ are in different components. But, since $S(p)$ and $S$ are contiguous, they must have a point in common along the spanning arc from $r$ to $t$. Since $r$ and $t$ cannot be elements of $S$ we see that $S \cap S(p) \cap$ int $S(q)$ is non-null. This implies that $S(q)$ and $S(p)$ have interior points in common which contradicts our assumption.

PRoof OF (b). If $S(p)$ and $S(q)$ are translates of $S$, in opposite directions, then $S$ and $S(p)$ are translates, in the same direction, of $S(q)$. If we let $U$ and $L$ denote respectively, the upper and lower lines of support of the configuration, we see that the union of a vertical ray upward from $U \cap S$, a vertical ray downward from $L \cap S$ and a span- 
ning arc in $S$ which joins them, will separate the interiors of $S(p)$ and $S(q)$. Therefore their intersection must either be null or consist entirely of points of contiguity. But if they are contiguous it follows from part (a) of the lemma that they have interior points in common, which is a contradiction.

We now construct a set of six translates of $S$ which are contiguous with $S$ and can be shown to satisfy the conclusions of our theorem.

Given any direction $\theta$ let the coordinate system, with center at an interior point of $S$, have the positive $x$-axis extend in the direction $\theta$. Let $S(a, 0)=S_{1}$ be a right translate of $S$ which is contiguous with $S$. (Such a translate exists by Lemma 1.) By Lemmas 6 and 7 there exists a translate $S(b, c)=S_{2}$, such that $c \neq 0$, which is contiguous with both $S$ and $S(a, 0)$. We will assume that $c>0$. The remaining four translates are $S(b-a, c)=S_{3}, S(-a, 0)=S_{4}, S(-b,-c)=S_{5}$ and $S(a-b,-c)=S_{6}$. By considering the various pairs of $S_{i}$ whose union with $S$ form congruent translates of $S \cup S_{1} \cup S_{2}$ it is apparent that all of the $S_{i}, i=1, \cdots, 6$, are contiguous to $S$.

The successor to $S_{i}$ is $S_{i+1}$ if $i \neq 6$ and $S_{1}$ if $i=6$. It will be denoted by $S_{i}^{\prime}$. The successor to $S_{i}^{\prime}$ is $S_{i}^{\prime \prime}$. The point $(x, y)$ is called the center of $S(x, y)$.

We note that, due to the manner in which we have constructed the set of six translates, the centers of $S_{i}$ and $S_{i}^{\prime \prime}$ are symmetric with respect to the midpoint of the line segment joining the center of $S_{i}^{\prime}$ to the origin.

\section{Lemma 8. The interiors of $S_{i}$ and $S_{i}^{\prime \prime}$ have no points in common.}

Proof. Rotate the configuration $S \cup S_{i} \cup S_{i}^{\prime} \cup S_{i}^{\prime \prime}$ so that the positive $x$-axis passes through the center of $S_{i}^{\prime}$. With this orientation the center of $S_{i}^{\prime \prime}$ will lie in the upper half plane and the center of $S_{i}$ in the lower half plane. Let $L$ and $R$ be the left hand and right hand lines of support for the configuration which are parallel to the line segment joining the centers of $S$ and $S_{i}$. Let $p$ be the point of $L \cap S$ with the largest ordinate and $q$ the point of $R \cap S_{i}^{\prime}$, with the smallest ordinate. If $t$ is a point of contiguity of $S$ and $S_{i}^{\prime}$, we see that the union of a horizontal ray extending to the left from $p$, a horizontal ray extending from $q$ to the right, a spanning arc from $p$ to $t$ in $S$ and a spanning arc from $t$ to $q$ in $S_{i}^{\prime}$, separates the plane so that interiors of $S_{i}$ and $S_{i}^{\prime \prime}$ lie in different components. This completes our proof.

It is now an easy matter to see that the preceding lemmas imply that the set of six translates which we have constructed satisfies the first four conclusions of our theorem. In order to show that it also satisfied the fifth conclusion we will have need of the following lemma. 
LEMMA 9. If $p$ and $q$ respectively, are points of contiguity of $S_{i}$ and $S_{i}^{\prime}$ with $S$, then they divide the boundary of $S$ into a closed arc $[p, q]$ and an open arc $(q, p)$ such that $[p, q]$ contains a point of contiguity with $S$ for each $S_{i}, i=1, \cdots, 6$, and $(q, p)$ contains no points of contiguity with $S$ for any $S_{i}$ except possibly for $S_{i}$ or $S_{i}^{\prime}$. (If $p=q$, then $(p, q)$ is the null set.)

Proof. Rotate the $x$-axis so that it is parallel to the line segment joining the centers of $S_{i}$ and $S_{i}^{\prime}$. From a highest point $r$ of $S_{i}$ construct a horizontal ray extending to the right and from the corresponding point $S$ of $S_{i}^{\prime}$ construct a horizontal ray extending to the left. The union of these rays together with a spanning arc from $r$ to $p$ in $S_{i}$, a spanning arc from $s$ to $q$ in $S_{i}^{\prime}$ and a spanning arc from $p$ to $q$ in $S$ clearly separates the plane so that all contiguous translates $S_{i}$, $i=1, \cdots, 6$, except $S_{i}$ and $S_{i}^{\prime}$ have their interiors in the lower component. It also separates the boundary of $S$ into arcs; one whose interior lies in the upper component and the other in the lower component. The open $\operatorname{arc}(q, p)$ in the upper half plane and the closed arc $[p, q]=\mathrm{bd} S-(q, p)$, have the desired properties.

For each $S_{i}$ pick a point $p_{i}$ which is a point of contiguity of $S_{i}$ with $S$. Let $\left[S_{i}, S_{i}^{\prime}\right]$ denote the union of $S_{i}, S_{i}^{\prime}$ and the open arc, whose existence is guaranteed by Lemma 9 , between the points of contiguity of $S_{i}$ and $S_{i}^{\prime}$ with $S$ which we have chosen.

We now complete the proof of our theorem by showing that $\bigcup_{i=1}^{6} S_{i}$ encloses $S$.

We need only prove that $\cup_{i=1}^{6} S_{i}$ separates any interior point of $S$ from infinity. Assume that it does not. From Lemma 9 we see that $\left[S_{1}, S_{2}\right]$ does not separate any interior point of $S$ from infinity. Since $\left[S_{1}, S_{2}\right]$ and $\bigcup_{i=1}^{6} S_{i}$ are both closed and their intersection which is $S_{1} \cup S_{2}$ is connected, it follows that their union does not separate any interior point of $S$ from infinity. Continuing this process we conclude that

$$
\bigcup_{i=1}^{6} S_{i} \cup \bigcup_{i=1}^{6}\left[S_{i}, S_{i}^{\prime}\right]
$$

does not separate any interior point of $S$ from infinity. But this union contains the boundary of $S$ which gives us a contradiction and thus concludes our proof.

3. Definition. A plane of support of a set $S$ in a linear space $L$ is the boundary of a minimal halfspace of $L$ which contains $S$. Two sets $A, B$ in $L$ are adjacent if they are on opposite sides of a common plane of support and their closures $\bar{A}, \bar{B}$ have a nonempty intersection. 
It is clear that adjacent closed sets are contiguous and that contiguous convex bodies are adjacent. In fact, if two sets are adjacent then so are their convex hulls.

THEOREM. Let $c_{n}$ be the maximal number of unit spheres in $E^{n}$ which can be adjacent to a given unit sphere without having interior points in common. Let $S$ be any bounded nonempty set in $E^{n}$, then there exist sets $S_{1}, S_{2}, \cdots, S_{c_{n}}$ with the following properties.

1. Each $S_{i}$ is the reflection of $S$ on a hyperplane of support $\pi_{i}$ of $S$ (and is therefore adjacent to $S$ ).

2. For $i \neq j$ the convex hulls, con $S_{i}$, con $S_{j}$, have no interior points in common.

3. The direction of $\pi_{1}$ may be prescribed arbitrarily, the direction of $\pi_{1} \cap \pi_{2}$ may be prescribed arbitrarily in $\pi_{1}$, and so on for all $\pi_{1} \cap \pi_{2}$ $\cap \cdots \cap \pi_{s}\left(s=1, \cdots, c_{n}\right)$.

REMARK. The number $c_{n}$ is known only for $n=1,2,3$ with $c_{1}=2$, $c_{2}=6$ and $c_{3}=12$. It would be interesting, but probably difficult, to find the general expression for $c_{n}$ or at least an asymptotic evaluation. We shall come back to this question later.

Proof. Consider a configuration $\mathfrak{F}$ in $E^{n}$ consisting of a unit sphere $U$ and unit spheres $U_{i}\left(i=1, \cdots, c_{n}\right)$ such that $U_{i}$ is tangent to $U$ at the point $p_{i}$ and $U_{i}, U_{j}$ have no interior points in common for $i \neq j$.

This configuration determines a polyhedron $P$ consisting of the intersection of the half-spaces $H_{i}$ which contain $U$ and have the plane of support to $U$ at $p_{i}$ as boundary. It is obvious that $P$ is convex and we shall now show that $P$ is bounded. If $P$ were unbounded then there would exist a half-line $l$ with vertex at the center $O$ of $U$ which lies entirely in the interior of $P$. Hence the angle which the line $O p_{i}$ makes with $l$ is nonacute. Thus all translates of $U$ in the direction of $l$ lie in $P$. In particular we get the translate $U^{\prime}$ whose center $O^{\prime}$ has distance 2 from $O$, so that $U^{\prime}$ is tangent to $U$ and has no interior points in common with any one of the $U_{i}$, contrary to the hypothesis that $c_{n}$ is maximal.

Two $(n-1)$-faces $F_{i}, F_{j}$ of $P$ are neighboring if they have an $(n-2)$-face of $P$ in common. If $F_{i}, F_{j}$ are neighboring $(n-1)$-faces of $P$ which are tangent to $U$ at $p_{i}, p_{j}$ respectively, then $\Varangle p_{i} O p_{j} \geqq \pi / 3$ since otherwise the spheres $U_{i}, U_{j}$ would have interior points in common.

Thus the reflections $P_{i}$ of $P$ on the planes of the $(n-1)$-faces $F_{i}$ of $P$ have no interior points in common.

Now let $S$ be any nonempty bounded set in $E^{n}$. In the usual man- 
ner we associate to every point $p$ of $U$ the plane of support to $S$ which is perpendicular to $O p$. We now construct the polyhedron $P^{\prime}$ whose face planes correspond to the points $p_{i}$ on $U$. (Not all the "face planes" need pass through $(n-1)$-faces of $P^{\prime}$.) The face angles of $P^{\prime}$ are the same as those of $P$ and hence the reflections of $P^{\prime}$ on its face planes will yield $c_{n}$ polyhedra $P_{i}^{\prime}$ which have no interior points in common. Since the closure $\bar{S}$ of $S$ meets every $P_{j}$ and since con $S \subseteq P^{\prime}$ our proof is complete except for part 3, which follows from the symmetry of the sphere.

We now give an estimate which shows $c_{n}$ to be of exponential growth.

TheOREM.

$$
\log \left(2(3)^{1 / 2} / 3\right) \leqq \lim \inf \frac{\log c_{n}}{n} \leqq \lim \sup \frac{\log c_{n}}{n} \leqq \log 2 .
$$

Proof. Using the notation in the proof of the preceding theorem we see that each $U_{i}$ subtends a cone at $O$ which intersects the surface $S$ of $U$ in a cap $C_{i}$ with center $p_{i}$ and geodetic radius $\pi / 6$. The hyperplane through the boundary of $C_{i}$ intersects $U$ in a solid $(n-1)$ dimensional sphere $S_{i}$ whose radius is $1 / 2$ and hence its $(n-1)$ measure is $m\left(S_{i}\right)=\pi^{(n-1) / 2} 2^{-(n-1)} / \Gamma((n+1) / 2)$.

Now $m\left(C_{i}\right)>m\left(S_{i}\right)$ and, since the $C_{i}$ have no interior points in common, $c_{n} m\left(C_{i}\right) \leqq m(S)$. Thus

$$
c_{n} \leqq \frac{m(S)}{m\left(C_{i}\right)}<\frac{n \pi^{n / 2} / \Gamma\left(\frac{n+2}{2}\right)}{\pi^{(n-1) / 2} 2^{-(n-1)} / \Gamma\left(\frac{n+1}{2}\right)},
$$

or

$$
\log c_{n}<n \log 2+O(\log n) .
$$

Hence $\lim \sup \log c_{n} / n \leqq \log 2$.

On the other hand, if we consider the caps $C_{i}^{\prime}$ with epicenter $p_{i}$ and geodesic radius $\pi / 3$ then their union must cover all of $S$; since any point $p$ in $S-U_{i} C_{i}^{\prime}$ would serve as point of tangency for an additional unit sphere which does not meet any of the $U_{i}$. Thus $c_{n} m\left(C_{i}^{\prime}\right) \geqq m(S)$ and hence

$$
c_{n} \geqq m(S) / m\left(C_{i}^{\prime}\right) .
$$

In order to estimate $m\left(C_{i}^{\prime}\right)$ let $S_{i}^{\prime}$ be the solid $(n-1)$-sphere whose boundary is that of $C_{i}^{\prime}$. Then $S_{i}^{\prime}$ has radius $3^{1 / 2} / 2$ and 


$$
m\left(S_{i}\right)=\pi^{(n-1) / 2}\left(\frac{3^{1 / 2}}{2}\right)^{n-1} / \Gamma\left(\frac{n+1}{2}\right) .
$$

Now $m\left(C_{i}^{\prime}\right)=\int_{s_{i}^{\prime}} \sec \gamma d m$ where $\gamma=\Varangle p_{i} O p$ at a point $p \in S_{i}^{\prime}$. Thus sec $\gamma \leqq 2$ and $m\left(C_{i}^{\prime}\right) \leqq 2 m\left(S_{i}^{\prime}\right)$. Substituting in $\left(^{*}\right)$ we obtain

$$
c_{n} \geqq m(S) / 2 m\left(S_{i}\right)=\frac{n \pi^{n / 2} / \Gamma\left(\frac{n+1}{2}\right)}{2 \pi^{(n-1) / 2}\left(3^{1 / 2} / 2\right)^{(n-1) / 2} / \Gamma\left(\frac{n+1}{2}\right)},
$$

$$
\log c_{n} \geqq n \log \left(2 \cdot 3^{1 / 2} / 3\right)+O(\log n) .
$$

Hence $\lim \inf \log c_{n} / n \geqq \log 2 \cdot 3^{1 / 2} / 3$.

This method of estimation can obviously be used to give exponential bounds for the maximal number of rays emanating from a point in $E^{n}$ so that the angle between any two rays is no less than $\alpha$.

Added January 23, 1959. Since this paper was written, B. Grünbaum has communicated to us several related results (his paper will appear in Pacific J. Math. under the title On a conjecture of $H$. Hadwiger). His discussion is restricted to convex bodies. He obtains the following results:

1. For a plane convex body $K$ the maximal number $M(K)$ of nonoverlapping contiguous translates is 6 when $K$ is not a parallelogram and 8 when it is.

2. In Euclidean $n$-space the $M(K)$ lies between $n^{2}+n$ (attained for the simplex) and $3^{n}-1$ (attained for the cube). Grünbaum conjectures that all even numbers between these limits and only these numbers can occur as $M(K)$.

This shows that the extremal property of the sphere, which we proved in $\S 3$, no longer holds if we restrict attention to translates rather than reflections and $n$ is sufficiently large.

3 . For a plane convex body $K$ the minimal number $m(K)$ of translates which do not overlap $K$ and surround it is 6 when $K$ is not a parallelogram and 4 when it is.

We proved that the upper bound holds for arbitrary compact Jordan regions. It seems likely that the lower bound also holds for such regions, although it does not hold in more general cases such as e.g. regular compact connected sets. It is easy to see that in higher dimensions $m(K)$ is not bounded from above for compact Jordan regions. The lower bound presumably is $2 n$; attained for the cube. For the unit sphere, $S$, the problem of determining $m(S)$ is that of determining the minimal number of spherical caps of geodetic radius $\pi / 3$ which will cover $S$. 


\section{REFERENCES}

1. G. T. Whyburn, Analytic topology, Amer. Math. Soc. Colloquium Publications, vol. 28, New York, 1942.

2. R. L. Wilder, Topology of manifolds, Amer. Math. Soc. Colloquium Publications, vol. 32, New York, 1949.

University of CALIFornia, Riverside,

RAND Corporation, Santa Monica,

Ramo-Wooldridge Corporation, Los ANGeles and

University of California, Los ANGeles 